\title{
Finite Element Algorithms for Computational Biomechanics of the Brain
}

\author{
Adam Wittek*, Grand Roman Joldes, Karol Miller \\ Intelligent Systems for Medicine Laboratory, \\ Department of Mechanical Engineering, \\ The University of Western Australia \\ 35 Stirling Highway, Crawley-Perth, 6009 Western Australia, \\ Australia \\ *Email: adam.wittek@uwa.edu.au
}

\section{Introduction}

Modelling of the brain biomechanical responses due to injury-causing transients and surgery is a problem of continuum mechanics that involves irregular geometry, complex loading and boundary conditions, non-linear materials, and large deformations (see Chapter 5 and Chapter 6). Finding a solution for such a problem requires computational algorithms of non-linear continuum mechanics.

As stated in Chapter 5, modelling for brain injury simulation has been driven by the idea that numerical surrogates of the human brain can be applied in the design of countermeasures mitigating the traumatic brain injury. Such modelling has been done with significant contribution and involvement of the automotive manufacturers [1] and participation of organisations responsible for traffic safety (e.g. National Highway Traffic Safety Administration NHTSA) [2]. Because of this industrial links and implications, modelling of the brain for injury simulation has been dominated by the explicit dynamics (i.e. utilising explicit time stepping referred to in the literature also as explicit time integration $[3,4]$ ) non-linear finite element algorithms available in commercial finite element codes, such as LS-DYNA [5], PAM-SAFE [6], RADIOSS [7], and ABAQUS [8], that are routinely used by the automotive industry.

In computational biomechanics for medicine, on the other hand, significant research effort has been directed to development of 
specialised algorithms that can provide the results within the real-time constraints of surgery. For instance, great interest was given to massspring method $[9,10]$ in which the analysed continuum is modelled as a discrete system of nodes (where the mass is concentrated) and springs. Due to its simplicity and low computational costs, the target application areas for this method included simulation software for virtual reality training systems for minimally invasive surgery [11] and surgical planning [12]. However, the behaviour of the mass-spring models strongly depends on the topology of the spring network. Furthermore, the spring parameters are difficult to identify and express in terms of soft tissue constitutive parameters (such as Young's modulus and Poisson's ratio) used in continuum mechanics [13]. Therefore, in recent years, more interest has been given to finite element method [4] that utilises the principles of continuum mechanics and does not suffer from the limitations of the mass-spring method.

Traditionally real-time computations for biomechanics for medicine relied on linear finite element algorithms that assume infinitesimally small deformations [13-19]. However, this assumption is not satisfied in surgical procedures where large deformations of the organ undergoing surgery occur. Examples include the brain deformations due to craniotomy (referred to in the literature as brain shift [20]) (see Figure 1 in Chapter 6) and needle insertion where the strain reaches over 0.8 [21] (Figure 1 below). Therefore, we focus on the algorithms that utilise fully non-linear (i.e. accounting for finite deformations and non-linear stress-strain relationships of soft tissues) formulation of solid mechanics and can be applied to any situation. In such formulation, current volume and surface of the modelled body organ, over which the integration of equations of continuum mechanics is to be conducted, are unknown: they are part of the solution rather than input data (Figure 2). The literature indicates that taking into account geometric non-linearity (through finite deformation formulation of equations of continuum mechanics) is needed to ensure accuracy of prediction of soft organ deformations even for applications that do not involve large strains (e.g. brain shift) [22]. 
a)

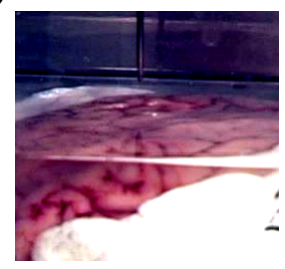

b)

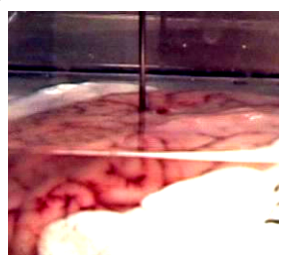

c)

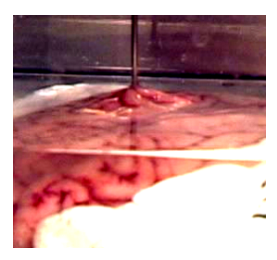

Figure 1 Swine brain deformation during needle insertion. a) Before insertion; b) During the insertion (note the deformation in the insertion area); c) During the needle removal. The experiments were conducted at the laboratory of the Surgical Assist Technology Group, Group, Institute for Human Science and Biomedical Engineering, National Institute of Advanced Industrial Science and Technology (AIST), Tsukuba, Ibaraki, Japan. For the experiment description see Wittek et al. [21].

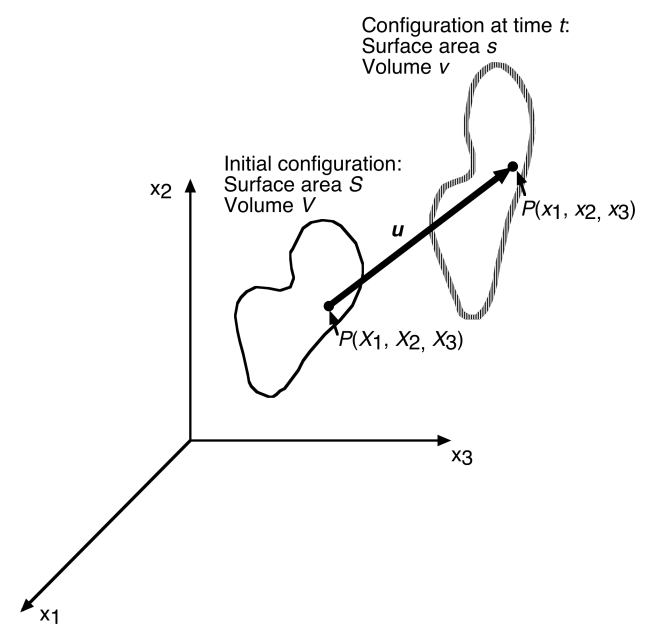

Figure 2 Computational biomechanics as a non-linear problem of continuum mechanics. Under transient load/impact and during surgery human body organs undergo large displacements (composed of rigid body motions and local deformations). Consequently, the equations of continuum mechanics governing the organ behaviour need to be integrated over the current surface $S$ and volume $V$. 
In the subsequent sections of this Chapter, we discuss the following topics:

- Section 2: Non-linear explicit dynamics finite element algorithms implemented in commercial finite element codes applied in modelling for brain injury biomechanics.

- Section 3: Specialised non-linear finite element algorithm for surgery simulation that utilises explicit time stepping and Total Lagrangian incremental formulation of continuum mechanics.

- Section 4: Specialised non-linear finite element algorithm that utilises Dynamic Relaxation and Total Lagrangian formulation for computation of steady state brain deformation within the realtime constraints of image-guided neurosurgery.

- Section 5: Element formulation for the specialised algorithms for surgery simulation and neurosurgery modelling, which includes non-locking tetrahedral element and efficient hourglass control for hexahedral element.

- Section 6: An efficient sliding contact algorithm for modelling of brain-skull interaction for image-guided neurosurgery.

- Section 7: Implementation of the specialised non-linear finite element algorithms for neurosurgery modelling on graphics processing units (GPUs) for real-time solution of the brain models for computer-assisted neurosurgery.

- Section 8: Verification of finite element algorithms of computational biomechanics.

\section{Algorithms for Injury Biomechanics}

Impact/injury biomechanics of the brain deals with events of very short duration (hundreds of milliseconds) in which the head is subjected to transient loads due to either direct impact or rapid acceleration that result in large deformation (or even mechanical damage) of the brain tissue. As indicated in [4, 23], non-linear finite element procedures with explicit time stepping outperform other algorithms in modelling of three-dimensional continua subjected to short-duration 
transient loads. Therefore, they have been a preferable choice in injury biomechanics [24] (see also Chapter 5) and are implemented in numerous finite elements codes (such as LS-DYNA, PAM-CRASH, ABAQUS, RADIOSS) industrially applied in impact injury simulation.

In impact injury simulation and other transient dynamics problems, the global system of finite element equations to be solved at each time step is:

$$
\mathbf{M} \ddot{\mathbf{u}}_{n}+\mathbf{K}\left(\mathbf{u}_{n}\right) \cdot \mathbf{u}_{n}=\mathbf{R}_{n},
$$

where $\mathbf{u}_{n}$ is a vector of nodal displacements at time step $n, \ddot{\mathbf{u}}_{n}$ is a vector of nodal acceleration at time step $n, \mathbf{M}$ is a mass matrix, $\mathbf{K}$ is a stiffness matrix non-linearly dependent on the deformation (because of the geometric and material non-linearities), $\mathbf{R}_{n}$ is a vector of nodal (active) forces at time step $n$, The product of the stiffness matrix and nodal displacements vector gives the nodal reaction forces vector $\mathbf{F}$. In explicit dynamics finite element procedures, the accelerations determined from equation of motion (Eq. 1) are integrated to calculate the displacements using the difference methods. Although many difference methods exist [4], the central difference method is the most commonly used due to its efficiency $[4,23]$ :

$$
\begin{aligned}
& u_{n+1}=u_{n}+\Delta t \cdot \dot{u}_{n}+1 / 2 \cdot \Delta t^{2} \cdot \ddot{u}_{n}, \\
& \dot{u}_{n+1}=\dot{u}_{n}+1 / 2 \cdot \Delta t \cdot\left(\ddot{u}_{n+1}+\ddot{u}_{n}\right),
\end{aligned}
$$

where $\Delta t$ is the time step (time increment).

Combining the central difference method given by Eq (2) and Eq. (3) with the global system of finite element equations Eq. (1) leads to the following formula for the vector of nodal displacements $\mathbf{u}_{n+1}$ at the increment $n+1$ when the time step $\Delta t$ is constant:

$$
\mathbf{M} \mathbf{u}_{n+1}=\Delta t^{2}\left(\mathbf{R}_{n}-\sum \mathbf{F}_{n}^{(i)}\right)-\mathbf{M}\left(\mathbf{u}_{n-1}-2 \mathbf{u}_{n}\right) \text {. }
$$

Formally Eq. (4) represents a system of equations. This system can be decoupled by using a lumped mass matrix $\mathbf{M}$. For lumped mass 
matrices, all non-diagonal components equal zero. Such lumped mass matrix corresponds to discretising the mass distribution by concentrating the mass at the nodes of each element. For such matrices, the system of equations Eq. (4) becomes an explicit formula for the unknown nodal displacements $\mathbf{u}_{n+1}$ :

$$
\mathbf{u}_{n+1}^{j}=\Delta t^{2}\left(\mathbf{R}_{n}^{j}-\sum_{i} \mathbf{F}_{n}^{(i) j}\right) / m_{j j}-\left(\mathbf{u}_{n-1}^{j}-2 \mathbf{u}_{n}^{j}\right),
$$

where $\mathbf{u}_{n+1}^{j}$ is a vector of nodal displacements at node $j$ at time step $n+1, m_{j j}$ is the component of the lumped mass matrix corresponding to node $j$ (i.e. mass lumped to node $j$ ), $\mathbf{R}_{n}^{j}$ is the vector of external forces applied to node $j, \sum_{i} \mathbf{F}_{n}^{(i) j}$ is the vector of nodal reaction forces at node $j$ (sum of contribution of the elements $i$ to which the node belong), and $\Delta t$ is the time step.

In Eq. (5), the computations are done at an element level eliminating the need for assembling the stiffness matrix of the entire analysed continuum. The mechanical properties of the analysed continuum are accounted for in the constitutive model and included in the calculation of nodal reaction forces $\mathbf{F}$. Thus, the computational cost of each time step and internal memory requirements are very low. It is worth noting that there is no need for iterations anywhere in the algorithm summarised in Eq. (5) even for non-linear problems. This implies the following advantages of non-linear finite element procedures utilising explicit time stepping and mass lumping:

- straightforward treatment of non-linearities without any need for iterations (no iterations required for a time step);

- no need to solve a system of equations;

- low computation cost for each time step;

- low internal memory requirements.

However, explicit time stepping methods are only conditionally stable. A restriction (Courant criterion [4]) on the time step size has to be included in order to obtain stable simulation results. The time step (referred to as a critical time step $\Delta t_{\text {critical }}$ ) that ensures the computation stability is equal to the smallest ratio of the characteristic 
length of an element divided by the dilatational (acoustic) wave speed in the element $[23,25,26]$ :

$$
\Delta t_{\text {critical }}=\left(\Delta t_{e}\right)_{\min } \leq\left(\frac{L_{e}}{c_{e}}\right)_{\text {min }},
$$

where is the characteristic element length $L_{e}$ and $C_{e}$ is the dilatational (acoustic) wave speed in the element. The formulae for calculating the characteristic element length $L_{e}$ for various commonly used finite elements are given in Belytschko [27].

For a model with the uniform properties, where the acoustic wave speed is the same in all the elements, Eq. (6) is equivalent to setting the condition that the time step cannot be longer than the travel time of the wave across the smallest element (i.e. the element with the smallest characteristic length $L_{e}$ ) in the mesh [23]. Based on [27], the acoustic wave speed can be expressed as

$$
c=\sqrt{\frac{E}{\rho} \frac{(1-v)}{(1+v)(1-2 v)}},
$$

where $E$ is the Young's modulus, $\rho$ is the density, and $v$ is the Poisson's ratio.

It should be noted that the stability limit given in Eq. (6) has been derived for linear problems. However, according to Belytschko [23], there is considerable empirical evidence that it can be also used for non-linear problems with an appropriate safety factor for the critical time step.

Eq. (6) and Eq. (7) imply that the critical time step can be increased by increasing the density (and hence mass) of the smallest elements (as determined by the characteristic length $L_{e}$ ) in the mesh. This process is referred to as mass scaling [5, 8]. Moderate mass scaling does not significantly change the responses of the analysed continuum and is regarded as a powerful method for decreasing the computation time [28]. However, it is also acknowledged that too severe scaling can introduce non-physical inertial effects [28]. Formal guidelines for determining permissible mass scaling limits for injury simulation have not been formulated yet and the quantitative information about the scaling used is rarely provided in the biomechanical literature. The limit of $5 \%$ of the total mass increase of the model due to 
mass scaling has been used by some authors [29]. However, Majumder et al. [30] reported a significant reduction in computation time for scaling resulting in the total model mass increase by as low as $0.016 \%$. For quasi-static problems, mass scaling resulting in local density increase by over 100 times, while simultaneously ensuring that the ratio of kinetic to elastic energy remains low, has been used in several studies [28, 31].

Stress calculation for obtaining the nodal forces $\mathbf{F}$ (see Eq. 4 and Eq. 8) is the major computation cost of the explicit dynamic finite element procedures summarised in Eq. (4) and Eq. (5). This implies that the complexity of the elements, which includes the number of nodes (element vertices), order of shape function polynomial, and the number of spatial integration points per element associated with the complexity are the key factors determining the number of computations in these procedures. Therefore, as mentioned in Chapter 5, in injury simulation utilising non-linear explicit dynamics finite element procedures, 8-noded hexahedron and 4-noded tetrahedron with linear shape functions and one integration (Gauss) point are the most commonly used elements $[32,33]$.

8 -noded hexahedron with one integration point is an underintegrated element (or low-order Gauss quadrature element), i.e. an element for which the stiffness matrix rank is lower than the number of element's degrees of freedom minus the number of rigid body modes [34]. Underintegrated elements exhibit instability known as hourglassing or zero-energy mode, i.e. nodal displacement vector which produces no strain energy but is not a rigid body motion [32, 34].

4-noded tetrahedral elements with linear shape functions and one Gauss point do not suffer from hourglassing. However, for incompressible (or nearly incompressible) continua, such as soft tissues, 4noded tetrahedral elements exhibit artificial stiffening known as volumetric locking [35]. A more detailed discussion on algorithms for hourglass control and volumetric locking reduction is provided in section 3. Algorithms for Surgery Simulation below. 


\section{Algorithms for Surgery Simulation}

As discussed in Chapter 6, surgical simulation systems are required to provide visual and haptic feedback to a surgeon or trainee. Such systems must provide time accurate prediction of the deformation field within an organ and interaction force between the surgical tool and the tissue at frequencies of at least $500 \mathrm{~Hz}$. From the perspective of continuum mechanics, such prediction requires solving the problem involving large deformations, non-linear constitutive properties and non-linear boundary conditions within very strict time constraints of haptic feedback.

For relatively slowly varying loads, such as those that occur due to interactions between the tissue and surgical tool, non-linear finite element algorithms utilising implicit time stepping are traditionally recommended in the literature for solving non-linear problems of solid mechanics [4]. Such procedures rely on solving systems of algebraic equations and require computationally expensive iterations. In contrast, the algorithms for injury simulation lead to an explicit formula for unknown nodal displacements (Eq. 5). For such algorithms, the number of operations per time step is typically three orders of magnitude smaller than for the algorithms relying on implicit time stepping [23]. Despite the fact that surgical simulations involve phenomena of duration of orders of magnitude longer than those that are of interest in injury simulation, the restrictions on the time step size (Courant criterion) required for solution stability (Eq. 6) do not compromise efficiency of explicit time stepping in such simulations. This is because the acoustic wave speed is proportional to square root of the analysed continuum Young's modulus (Eq. 7) which is very low (under $10^{4} \mathrm{~Pa}$ ) for the brain tissue. For instance, in the simulation of needle insertion into the brain conducted using non-linear explicit dynamics finite element procedures reported in Wittek et al. [21], the time step was over $1.5 \cdot 10^{-2} \mathrm{~ms}$. In contrast, in typical engineering applications, such as metal forming, the integration steps are of an order of $10^{-5} \mathrm{~ms}[36]$.

In commercial finite element codes utilising explicit time stepping, the calculated variables (such as displacement, strain and stress) are incremented by referring them to the current configuration of the 
analysed continuum, which is known as an Updated Lagrangian formulation [4]. However, we advocate a Total Lagrangian (TL) formulation of computational mechanics in which all variables are referred to the original configuration of the system [37]. The decisive advantage of this formulation is that all derivatives with respect to spatial coordinates are calculated with respect to the original configuration and therefore can be pre-computed as shown in the flowchart of the Total Lagrangian Explicit Dynamics (TLED) algorithm presented in Figure 3 (a detailed description of the algorithm is given in Miller et al. [37]). In Chapter 11), the application of Total Lagrangian formulation with explicit time stepping is extended beyond finite element analysis to meshless methods of computational biomechanics that provide stable solution in a presence of discontinuities/cracks due to surgical dissection and tissue rupture and allow for semi-automated generation of patient-specific computational grids directly from medical images.

Following the approach typically applied in explicit dynamics finite element analysis, for computational efficiency of our TLED algorithm, we used single point spatial integration for all elements of the mesh (improved linear tetrahedrons [38] and under-integrated linear hexahedrons). Therefore, the nodal forces for each element are computed as:

$$
{ }_{0}^{t} \mathbf{F}_{\text {int }}={ }_{0}^{t} \mathbf{X} \cdot{ }_{0}^{t} \mathbf{S} \cdot \mathbf{B}_{0} \cdot V_{0},
$$

where according to the notation used in Bathe [4], the left superscript represents the current time, the left subscript represents the time of the reference configuration, $\mathbf{F}_{\text {int }}$ is the matrix of nodal forces, $\mathbf{B}_{0}$ is the matrix of shape function derivatives, $\mathbf{S}$ is the second Piola Kirchoff stress matrix, $\mathbf{X}$ is the deformation gradient and $V_{0}$ is the initial volume. In Eq. (8), the matrix of shape function derivatives $\mathbf{B}_{0}$ and the initial volume $V_{0}$ are constant and therefore can be pre-computed (see Figure 3).

The main benefits of the TLED algorithm in comparison to the explicit dynamics algorithms using Updated Lagrangian formulation are:

- Allows pre-computing of many variables involved (e.g. derivatives with respect to spatial coordinates), Figure 3; 
- No accumulation of errors - increased stability for quasi-static solutions;

- Second-Piola Kirchoff stress and Green strain are used - appropriate for handling geometric non-linearities;

- Easy implementation of the material law for hyper-elastic materials using the deformation gradient.

The fact that many quantities involved in the computation of nodal forces can be pre-computed leads to a significant decrease in the computational effort. For instance, the TLED algorithm using eight-noded hexahedral under-integrated elements requires approximately $35 \%$ fewer floating-point operations per element, per time step than the Updated Lagrangian explicit algorithm using the same elements [37].

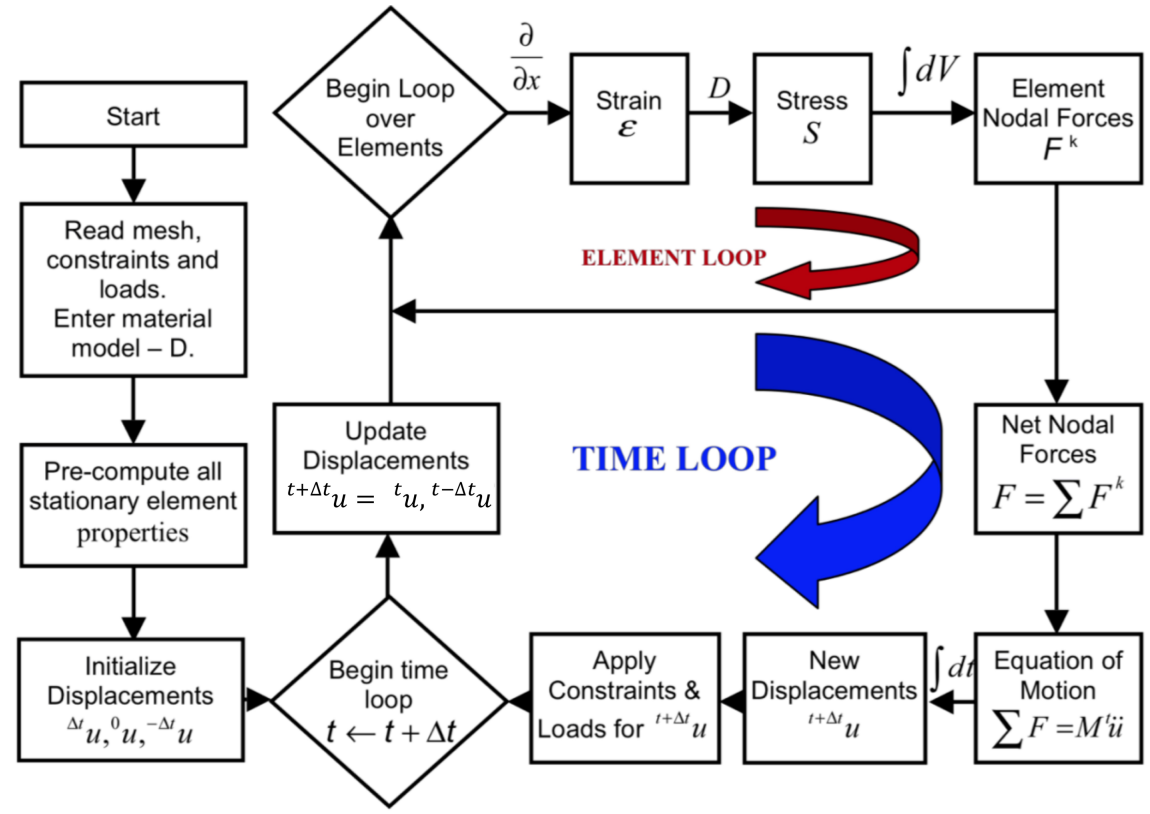

Figure 3 Flow-chart of the Total Lagrangian Explicit Dynamics (TLED) finite element algorithm for surgery simulation. Detailed description of the algorithm is given in Miller et al. [37]. 


\section{Algorithms for Neurosurgery Modelling}

As explained in Chapter 6 accurate warping of high-quality pre-operative radiographic images to the intra-operative (i.e. deformed) brain configuration in a process known as non-rigid registration is a key element of image-guided neurosurgery. In order to perform such warping, only the final (deformed during surgery) state of the brain needs to be predicted. This requires algorithms for determining steady state solution for the brain deformations. The deformations at the steady state must be obtained within the real-time constraints of image-guided neurosurgery, which practically means that the results should be available in 40-80 seconds.

As stated in Chapter 6, the neuroimage registration is a non-linear problem of computational mechanics as it involves large deformations, non-linear material properties and non-linear boundary conditions. However, it is a less demanding problem than surgery simulation as only the steady state solution for deformations is of interest, i.e. the time history of forces and deformations does not have to be obtained. Therefore, for image registration, we advocate combining Total Lagrangian (TL) formulation, discussed in section 3. Algorithms for Surgery Simulation, with Dynamic Relaxation (DR) which is an explicit iterative algorithm that relies on introduction of an artificial mass dependent damping term in the equation of motion. The damping attenuates the oscillations in the transient response, increasing the speed of convergence towards the steady state solution. Because DR is an explicit algorithm, there is no need for solving large systems of equations. All quantities can be treated as vectors, reducing the implementation complexity and the memory requirements. Although the number of iterations to obtain convergence can be quite large, the computation cost of each iteration is very low, making it a very efficient solution method for non-linear problems.

\subsection{Dynamic Relaxation algorithm}

The basic Dynamic Relaxation (DR) algorithm is presented in [39]. The main idea is the inclusion of a mass proportional damping in 
equation of motion (Eq. 1) (see section 2. Algorithms for Injury Simulation), which increases the convergence speed towards the steady state, and then solving the obtained damped equation using the central difference method (explicit time stepping). After the inclusion of mass proportional damping, equation of motion (Eq. 1) becomes

$$
\mathbf{M} \ddot{\mathbf{u}}+c \mathbf{M} \dot{\mathbf{u}}+\mathbf{K}\left(\mathbf{u}_{n}\right) \cdot \mathbf{u}_{n}=\mathbf{R}_{n},
$$

where $C$ is the damping coefficient.

By applying the central difference integration method to the damped equation of motion (Eq. 9), the equation that describes the iterations in terms of displacements becomes:

$$
\begin{aligned}
& \mathbf{u}_{n+1}=\mathbf{u}_{n}+\beta\left(\mathbf{u}_{n}-\mathbf{u}_{n-1}\right)+\alpha \mathbf{M}^{-1}(\mathbf{R}-\mathbf{F}), \\
& \alpha=2 \Delta t^{2} /(2+c \Delta t), \quad \beta=(2-c \Delta t) /(2+c \Delta t),
\end{aligned}
$$

where $\Delta t$ is length of the time increment (time step).

The iterative method defined by Eq. (10) is explicit as long as the mass matrix is diagonal. As the mass matrix does not influence the deformed state solution, a lumped scaled mass matrix can be used that maximises the convergence of the method.

In Underwood [39], the convergence of the DR algorithm is studied for linear structural mechanics equations, when the nodal forces can be written as

$$
\mathbf{F}(\mathbf{u})=\mathbf{K} \cdot \mathbf{u}
$$

where $\mathbf{K}$ is the stiffness matrix.

We extend this study to the non-linear case. We propose to use the linearisation of the nodal forces obtained by expanding them in a Taylor series and keeping the first two terms

$$
\mathbf{F}(\mathbf{u})=\mathbf{F}\left(\mathbf{u}_{k}\right)+\mathbf{K}_{k} \cdot\left(\mathbf{u}_{n}-\mathbf{u}_{k}\right)
$$

where $\mathbf{u}_{k}$ is a point close to $\mathbf{u}_{n}$ and $\mathbf{K}_{k}$ is the tangent stiffness matrix evaluated at point $\mathbf{u}_{k}$.

By substituting $\mathbf{F}$ in Eq. (10) and Eq. (13), we obtain the equation that advances to a new iteration for a non-linear problem as:

$$
\mathbf{u}_{n+1}=\mathbf{u}_{n}+\beta\left(\mathbf{u}_{n}-\mathbf{u}_{n-1}\right)+\alpha \mathbf{b}-\alpha \mathbf{A} \mathbf{u}_{n},
$$


with

$$
\mathbf{b}=\mathbf{M}^{-1}\left(\mathbf{R}-\mathbf{F}\left(\mathbf{u}_{k}\right)+\mathbf{K}_{k} \mathbf{u}_{k}\right), \quad \mathbf{A}=\mathbf{M}^{-1} \mathbf{K}_{k} .
$$

It is worth noting that, even if Eq. (14) has the same form as in the linear case, the point $\mathbf{u}_{k}$ in Eq. (15) is not fixed during the iteration process (as it must be close to $\mathbf{u}_{n}$ in order for the Taylor series expansion to be accurate) and therefore the tangent stiffness matrix (and matrix $\mathbf{A}$ ) changes.

The error after the $n_{\text {th }}$ iteration is defined as:

$$
\mathbf{e}_{n}=\mathbf{u}_{n}-\mathbf{u}^{*}
$$

where $\mathbf{u}^{*}$ is the solution. Substituting Eq. (16) in Eq. (14) gives the error equation (valid only close to the solution):

$$
\mathbf{e}_{n+1}=\mathbf{e}_{n}-\alpha \mathbf{A} \mathbf{e}_{n}+\beta\left(\mathbf{e}_{n}-\mathbf{e}_{n-1}\right) .
$$

By assuming that

$$
\mathbf{e}_{n+1}=\mathbf{K} \cdot \mathbf{e}_{n},
$$

the following relation is obtained for computing the eigenvalues $\kappa$ of matrix $\mathbf{~}$ :

$$
\kappa^{2}-(1+\beta-\alpha A) \kappa+\beta=0
$$

where $A$ denotes any eigenvalue of matrix $\mathbf{A}$.

The fastest convergence is obtained for the smallest possible spectral radius $\rho=|\mathbf{k}|$. The optimum convergence condition is obtained when:

$$
\begin{aligned}
& \rho^{*}=\left|\kappa^{*}\right|=\beta^{1 / 2} \approx\left|1-2 \sqrt{\frac{A_{0}}{A_{m}}}\right|, \\
& \Delta t \approx 2 / \sqrt{A_{m}}=2 / \omega_{\max }, \\
& c \approx 2 \sqrt{A_{0}}=2 \omega_{0},
\end{aligned}
$$

where $A_{0}$ and $A_{m}$ are the minimum and maximum eigenvalues of matrix $\mathbf{A}$ and therefore $\omega_{0}$ and $\omega_{\max }$ are the lowest and highest circular frequencies of the un-damped equation of motion [39]. 
The effect of eigenvalue estimation accuracy on the convergence of the method is presented in Joldes et al. [40]. To ensure convergence, it is critical that the maximum eigenvalue $A_{m}$ is over-estimated, even if this will lead to a decreased convergence speed. If, at the same time, the minimum eigenvalue $A_{0}$ is under-estimated, uniform convergence will be obtained for all eigenvalues, with a further decrease in the convergence speed. If $A_{0}$ is over-estimated, then it is possible to increase the convergence speed for all eigenvalues except a very narrow range of small eigenvalues.

\subsubsection{Dynamic Relaxation algorithm: Maximum eigenvalue $A_{\mathrm{m}}$ and mass matrix}

Using the Gerschgorin's theorem [25, 41], it has been demonstrated that the maximum eigenvalue of an assembled finite element mesh is bounded by the maximum eigenvalue of any of the elements in the mesh [4]:

$$
A_{m} \leq \max _{e}\left(\lambda_{\max }^{e}\right) .
$$

Therefore, an estimation of the maximum eigenvalue can be obtained by estimating the maximum eigenvalue of each element in the mesh. Such estimations for different element types are presented in Hughes [26].

In the case of a non-linear problem, the maximum eigenvalue changes during the simulation as the geometry of the elements changes and therefore it must be estimated after every iteration step.

Because the mass matrix has no influence on the steady state (as the time derivatives in Eq. 9 become zero), a fictitious mass matrix that improves the convergence rate can be used. The mass matrix can be chosen such that it reduces the condition number of matrix $\mathbf{A}$, leading to a decrease in the spectral radius $\rho$ (see Eq. 20).

In order to reduce the condition number we propose to align the maximum eigenvalue of all elements in the mesh to the same value by changing the density of each element. By doing this we can still use Eq. (23) for estimating the maximum eigenvalue, and the condition number is at least preserved and generally decreased, as shown in Underwood [39]. This process guarantees that the selected 
maximum eigenvalue $A_{m}$ is an over-estimation of the actual maximum eigenvalue during the simulation, therefore ensuring the convergence.

\subsubsection{Dynamic Relaxation algorithm: Estimation of the minimum eigenvalue $A_{0}$}

Estimating the minimum eigenvalue is a challenging task, especially for non-linear problems, where an adaptive procedure should be used in order to obtain the optimum convergence parameters. An overview of the procedures proposed by different authors in the context of DR (including an adaptive one) is presented in Underwood [39].

The adaptive method proposed in Underwood [39] is based on Rayleigh's quotient and the use of a local diagonal stiffness matrix. The elements of this matrix are computed using finite differences, which can be very difficult to do for degrees of freedom which have small displacement variation.

In this section we propose a new adaptive method for computing the minimum eigenvalue, which is also based on Rayleigh's quotient, but does not have the shortcomings of the method proposed in [39].

We consider a change of variable:

$$
\mathbf{z}_{n}=\mathbf{u}_{n}-\mathbf{u}_{k},
$$

where $\mathbf{u}_{k}$ is the point used for linearisation of the nodal forces in Eq. (13). The linearised nodal forces can therefore be expressed as:

$$
\mathbf{F}\left(\mathbf{u}_{n}\right)=\mathbf{F}\left(\mathbf{u}_{k}\right)+\mathbf{K}_{k} \cdot \mathbf{z}_{n},
$$

and the linearised equation of motion will become, by replacing Eq. (24) and Eq. (25) in Eq. (1):

$$
\mathbf{M} \cdot \ddot{\mathbf{z}}+\mathbf{K}_{k} \cdot \mathbf{z}=\mathbf{R}-\mathbf{F}\left(\mathbf{u}_{k}\right) \text {. }
$$

We can now rely on Eq. (26) to estimate $A_{0}$ using Rayleigh's quotient and the current value of the displacements:

$$
A_{0} \leq \frac{\left(\mathbf{z}_{n}\right)^{T} \mathbf{K}_{k} \mathbf{z}_{n}}{\left(\mathbf{z}_{n}\right)^{T} \mathbf{M} \mathbf{z}_{n}}
$$


We consider the right hand side of Eq. (27) as an estimate of the minimum eigenvalue. Using Eq. (24) and Eq. (25), this estimate becomes [42]:

$$
A_{0} \approx \frac{\left(\mathbf{u}_{n}-\mathbf{u}_{k}\right)^{T}\left(\mathbf{F}\left(\mathbf{u}_{n}\right)-\mathbf{F}\left(\mathbf{u}_{k}\right)\right)}{\left(\mathbf{u}_{n}-\mathbf{u}_{k}\right)^{T} \mathbf{M}\left(\mathbf{u}_{n}-\mathbf{u}_{k}\right)},
$$

where $\mathbf{u}_{k}$ is a fix point that must be close to $\mathbf{u}_{n}$. We will choose the solution from a previous iteration as $\mathbf{u}_{k}$, and this point will be updated after a number of steps in order to keep it close to the current solution $\mathbf{u}_{n}$. No additional information (such as estimates of the stiffness matrix) is required and only vector operations are performed (as $\mathbf{M}$ is a diagonal lumped mass matrix).

During the iterative dynamic relaxation DR procedure, the high frequencies are damped out and the system will eventually oscillate at its lowest frequency. Therefore, Eq. (28) will converge towards the minimum eigenvalue. This estimation process, combined with our parameter selection process, leads to an increased convergence rate, because it always offers an over-estimation of the minimum eigenvalue. The higher the over-estimation of the minimum eigenvalue, the higher the reduction of the high frequency vibrations (see [40] and [42]), and therefore Eq. (28) will convergence faster towards the real minimum eigenvalue.

\subsubsection{Dynamic Relaxation algorithm: Termination criteria}

One very important aspect of any finite element (FE) algorithm is the termination criterion used. If the criterion is too coarse, then the solution might be too inaccurate and if the criterion is too tight, then time is lost for unnecessary computations.

The usual criteria used by FE software are based on residual forces, displacements or energy. None of these criteria gives any information about the absolute error in the solution and selecting any of these termination criteria is very difficult.

We propose a new termination criterion that gives information about the absolute error in the solution, particularly suited for our solution method. Because DR iterations lead to a strong reduction of the high frequencies, the displacement vector will oscillate around the 
solution vector with a frequency that converges towards the smallest oscillation frequency. That implies that the error vector $\mathbf{e}$ will converge toward the eigenvector corresponding to the lowest eigenvalue. Therefore, we can make the following approximation:

$$
\mathbf{A} \cdot \mathbf{e}_{n}=A_{0} \cdot \mathbf{e}_{n} .
$$

By substituting Eq. (29) in Eq. (17), and considering relations given in Eq. (19) and Eq. (20) we obtain:

$$
\mathbf{u}_{n+1}-\mathbf{u}^{*} \approx \rho\left(\mathbf{u}_{n}-\mathbf{u}^{*}\right),
$$

where $\mathbf{u}^{*}$ is the solution.

Therefore, after each iteration step the error is reduced by a ratio equal to $\rho$. We can now obtain an approximation of the absolute error in the solution by applying the infinity norm to Eq. (30):

$$
\begin{aligned}
& \left\|\mathbf{u}_{n+1}-\mathbf{u} *\right\|_{\infty} \approx \rho \cdot\left\|\mathbf{u}_{n}-\mathbf{u} *\right\|_{\infty} \leq \rho \cdot\left(\left\|\mathbf{u}_{n+1}-\mathbf{u} *\right\|_{\infty}+\left\|\mathbf{u}_{n+1}-\mathbf{u}_{n}\right\|_{\infty}\right), \\
& \left\|\mathbf{u}_{n+1}-\mathbf{u} *\right\|_{\infty} \leq \frac{\rho}{1-\rho} \cdot\left\|\mathbf{u}_{n+1}-\mathbf{u}_{n}\right\|_{\infty} .
\end{aligned}
$$

Therefore, the convergence criterion can be defined as:

$$
\frac{\rho}{1-\rho} \cdot\left\|\mathbf{u}_{n+1}-\mathbf{u}_{n}\right\|_{\infty} \leq \varepsilon,
$$

where $\varepsilon$ is the imposed absolute accuracy. This convergence criterion gives an approximation of the absolute error based on the displacement variation norm from the current iteration.

Because our parameter estimation procedures over-estimate the maximum eigenvalue $A_{m}$ and under-estimate the minimum eigenvalue $A_{0}$, the value of the computed spectral radius $\rho_{\mathrm{c}}$ we can use in Eq. (33) is lower than the real value of the spectral radius (see Eq. 20). This can lead to an early termination of the iteration process. Therefore, in Eq. (33) we use a corrected value of the computed spectral radius:

$$
\rho_{\infty}=\rho_{c}+\varsigma \cdot\left(1-\rho_{c}\right)
$$

where $\zeta$ is a correction parameter with values between 0 and 1 , defining the maximum under-estimation error for the spectral radius $\rho$. In our simulations we use $\zeta=0.2$. 


\section{Element Formulation for Finite Element Algorithms for Surgery Simulation and Neurosurgery Modelling}

\subsection{Volumetric locking}

As stated in Chapter 6, due to stringent computation time requirements, the finite element meshes for models applied in surgery simulation and image registration must be constructed using low order elements that are computationally inexpensive. Mixed meshes consisting of tetrahedral and hexahedral element are most convenient from the perspective of automation of simulation process. However, the standard formulation of the tetrahedral element exhibits volumetric locking, especially in case of soft tissues such as the brain, which are modelled as almost incompressible materials [43-49]. There is a number of improved linear tetrahedral elements already proposed by different authors [50-53]. The averaged nodal pressure (ANP) tetrahedral element proposed by Bonet and Burton in [50] is computationally inexpensive and provides much better results for nearly incompressible materials than the standard tetrahedral element. Nevertheless, one problem with the ANP element and its implementation in a finite element code is the handling of interfaces between different materials. In Joldes et al. [54], we extended the formulation of the ANP element so that all elements in a mesh are treated in a similar way, requiring no special handling of the interface elements.

\subsection{Stability of under-integrated hexahedral elements: Hourglassing}

As stated in Chapter 6, low-order hexahedral elements with one Gauss point (also referred to as a linear under-integrated hexahedral elements) are the preferred choice for explicit dynamics-type algorithms from the perspective of computational efficiency. However, such elements exhibit unphysical zero energy deformation modes (hourglass). The hourglass modes can be controlled by calculating hourglass forces that oppose the hourglass deformation modes. We have 
shown in Joldes et al. [55] that the hourglass control forces for each element can be computed (in matrix form) as:

$$
{ }_{0}^{t} \mathbf{F}^{\mathrm{Hg}}=k \cdot{ }_{0} \mathbf{Y} \cdot{ }_{0} \mathbf{Y}^{T} \cdot{ }_{0}^{t} \mathbf{u},
$$

where $k$ is a constant that depends on the element geometry and material properties, ${ }_{0} \mathbf{Y}$ is the matrix of hourglass shape vectors and $\mathbf{u}$ is the matrix of current displacements. As we use Total Lagrangian formulation TL, all quantities except $\mathbf{u}$ in Eq. (35) are constant and can be pre-computed. This makes the hourglass control mechanism very efficient.

\section{Modelling of the Brain - Skull Interactions for Image-Guided Neurosurgery: Efficient Finite Sliding Contact Algorithm}

Modelling of interactions between continua (e.g. soft organs) undergoing deformations is a challenging task. To facilitate such modelling, many sophisticated contact algorithms have been proposed in the literature (e.g. [56], [57], [58], [59]) and implemented in commercial finite element codes such as ABAQUS [8] [60] and LS-DYNA [5]. Application of such algorithms tends to consume significant computing resources, which substantially increases the solution time.

When computing the brain deformation for neuroimage registration, we are interested in the interactions between the brain and rigid skull that provide constraints for the brain tissue deformation and brain rigid body motion. Accurate modelling of such interactions can be done using a very efficient algorithm that treats these interactions as a finite sliding, frictionless contact between a deformable object (the brain) and a rigid surface (the skull) [61]. The main parts of such contact algorithm (for detailed description see [61]) are: detection of nodes on the brain surface (also called the slave surface) which have penetrated the skull surface (master surface) and the repositioning of each slave node that has penetrated the master surface to the closest point on the master surface.

Efficient penetration detection algorithm can be formulated based on the closest master node (nearest neighbour) approach [5]. As the surfaces of the anatomical structures of the segmented 
neuroimages are typically discretised using triangles, the skull surface can be treated as a triangular mesh. We refer to each triangle surface as a "face", to the vertices - "nodes" and to the triangle sides - "edges". Using this terminology, the basic brain-skull contact algorithm is described as follows:

- For each slave node P:

- Find the closest master node C (global search);

- Check the faces and edges surrounding $\mathrm{C}$ for penetration (local search);

- Check additional faces and edges that might be penetrated by $\mathrm{P}$ (identified in the master surface analysis stage - because the master surface is rigid this analysis can be done pre-operatively).

Following [56] and [59], further improvement of efficiency of the penetration detection algorithm and computation speed is done by implementing bucket sort in the global search phase.

\section{Real-Time Computations Without Supercomputers: Increasing Computation Speed Through Algorithm Implementation on Graphics Processing Unit (GPU)}

The algorithms for surgery simulation and image-guided surgery discussed in this Chapter facilitate efficient and robust computations. For instance, they make it possible to compute deformation field within the brain for image registration problem in under $40 \mathrm{~s}$ on a standard personal computer for non-linear finite element models consisting of around 30000 elements. However, predicting the time history of force between the soft tissue and surgical tool at frequency of $500 \mathrm{~Hz}$ required for haptic feedback poses a challenge even for very efficient non-linear algorithms deployed on a personal computer.

For hardware-based increase of computation speed, we advocate the implementation of the algorithms on graphics processing units (GPUs) by using a GPU as a coprocessor for the computer central processing unit (CPU) for executing sections of the code that can run 
in parallel. GPUs have a highly parallel, multithreaded, multicore processor architecture and its cost (under US\$ 3000 for most general purpose GPUs) is orders of magnitude smaller than that of a supercomputer with a comparable number of parallel threads. GPU architecture is well suited for problems that can be expressed as data-parallel computations with high arithmetic intensity, where the same program is executed on many data elements in parallel.

Before the introduction of NVIDIA's Compute Unified Device Architecture (CUDA) [62] and Open Computing Language (OpenCL) [63], general purpose computations on GPUs were done by recasting the computations in graphic terms and using the graphics pipeline. Therefore, a scientific or general-purpose computation often required a concerted effort by experts in both computer graphics and in the particular scientific or engineering domain. With the introduction of CUDA, in November 2006, NVIDIA proposed a new parallel programming model and instruction set for their GPUs that can be used for performing general-purpose computations. CUDA comes with a software environment that allows developers to use $\mathrm{C}$ as a high level programming language. A minimum set of keywords are used to extent the $\mathrm{C}$ language in order to: identify the code that must be run on the GPU as parallel threads, identify each thread (and the block of threads it belongs to) and to organise and transfer the data in the different GPU memory spaces. CUDA also exposes the internal architecture of the GPU and allows direct access to its internal resources facilitating the development of application software that transparently scales with the number of processor cores in the GPU. The programmer has more control over the internal hardware resources of the GPU, but this comes at the expense of an increased programming effort compared to a CPU implementation.

Because it only uses vectors, an explicit time stepping algorithm is perfectly suited for parallel implementation on GPU. We implemented the Dynamic Relaxation algorithm presented in section 4. Algorithms for Neurosurgery Modelling on GPU using CUDA. We transferred all the computationally intensive parts of the algorithm (element force computation, displacement vector computation, contact handling, parallel reduction - including infinity norm computation and scalar product of vectors) to the GPU, to take advantage of its massive parallelism. The code was run on a NVIDIA Tesla C870 
computing board, which has 16 multiprocessors with eight scalar processor cores each (i.e., 128 cores in total) and single-precision floating point operations. A detailed description of the implementation can be found in Joldes et al. [64]. The GPU implementation performs 2000 iterations of the brain shift simulation in $1.8 \mathrm{~s}$, offering real time computation capabilities. Other examples of GPU implementation of finite element algorithms of computational biomechanics that utilise TLED include widely used Simulation Open Framework Architecture (SOFA) $[65,66]$.

\section{Verification of Finite Element Algorithms of Computational Biomechanics}

The general guidelines for verification in computational solid mechanics have been proposed by the American Society of Mechanical Engineers ASME in [67]. These guidelines were preceded by the extensive discussion of the concepts of verification and validation in computational mechanics by Babuska and Oden [68]. They underscore the importance of establishing confidence through collection of evidence that the solution algorithms are working correctly. As for non-linear problems of computational solid mechanics analytical solutions typically do not exist, we advocate collecting such evidence by comparing the results obtained by means of new algorithms with the solutions from established algorithms such as those implemented in commercial finite element codes.

In the following sections we will present verification results for some of the algorithm described in this Chapter: hourglass control, volumetric locking, dynamic relaxation and brain-skull interaction (contact).

\subsection{Hourglass control}

The verification experiment was artificially designed to compound difficulties associated with hourglass control: large deformations, bending and rigid body motions. A column having a height of $1 \mathrm{~m}$ 
and a square section with the side size $0.1 \mathrm{~m}$ was meshed using hexahedral elements (Figure 4a). The mesh has 496 nodes and 270 elements. A neo-Hookean almost incompressible material model was used, having the mechanical properties similar to those of the brain (mass density of $1000 \mathrm{~kg} / \mathrm{m}^{3}$, Young's modulus in un-deformed state equal to $3000 \mathrm{~Pa}$ and Poisson's ratio 0.49).

The deformation was imposed by constraining the lower face and displacing the upper face of the column, with maximum displacements of $0.5 \mathrm{~m}$ in the $\mathrm{x}$ direction and $0.3 \mathrm{~m}$ in the $\mathrm{z}$ direction.

The deformed shape obtained using the TLED algorithm is presented in Figure $4 \mathrm{~b}$ for the under-integrated hexahedral elements with no hourglass control. The influence of the presented hourglass control mechanism can be clearly seen in Figure 4c.

The displacements of a line of nodes from the side of the column (in the plane $y=0$ ) is presented in Figure 5. These displacements are compared with the results obtained using the commercial finite element software ABAQUS (fully integrated linear hexahedral elements with hybrid displacement-pressure formulation).

The displacement maximum relative error, defined as the ratio between the maximum displacement difference and the imposed displacement, was $1.4 \%$ in case of column deformation. This demonstrates the good accuracy of the elements using the proposed hourglass control mechanism.

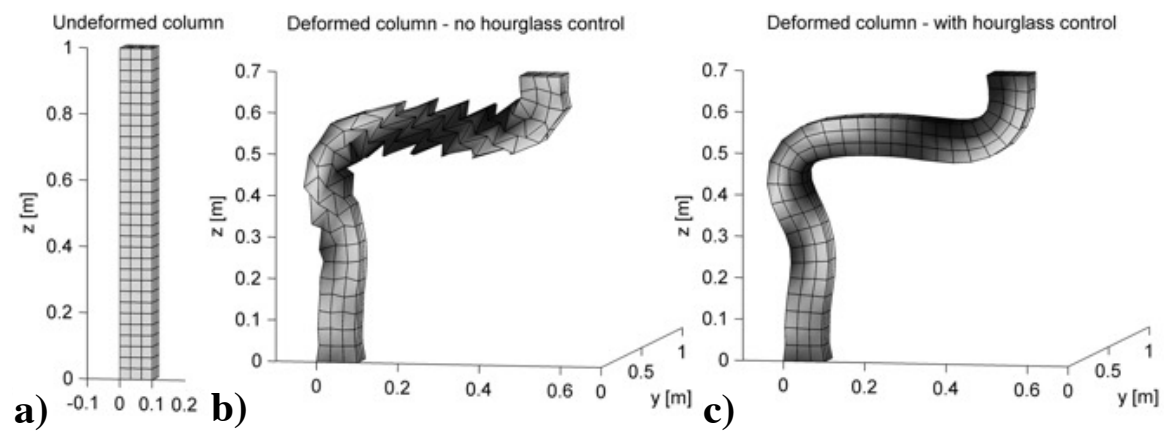

Figure 4 Verification of hourglass control algorithm using deformation of a column as an example. a) Undeformed shape; b) Deformed shape with no hourglass control (b); and c) Deformed shape with successful hourglass control. Adapted from Joldes et al. [55]. 


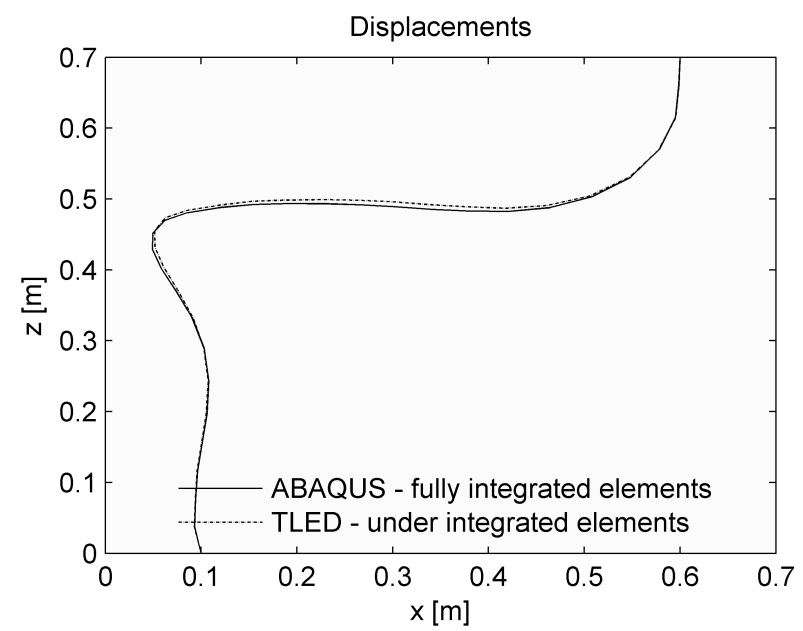

Figure 5 Deformation of a column - middle line displacements comparison with results from the commercial finite element software ABAQUS (fully integrated linear hexahedral elements with hybrid displacement-pressure formulation). Copied from Joldes et al. [55].

\subsection{Volumetric locking}

Because the only difference between our Improved Averaged Nodal Pressure (IANP) element and the standard Averaged Nodal Pressure ANP element consists in the way interfaces between different materials are handled, we designed a simulation experiment that highlights these differences. We considered a cylinder with a diameter of $0.1 \mathrm{~m}$ and a height of $0.2 \mathrm{~m}$ made out of alternating sections with two different material properties, as shown in Table 1. We used a neoHookean constitutive model for both materials. 
Table 1. Material properties

\begin{tabular}{|c|c|c|}
\hline Property & Material 1 & Material 2 \\
\hline Young's modulus E [Pa] & 3000 & 30000 \\
\hline Poisson ratio v & 0.49 & 0.48 \\
\hline Density @ [kg/m] & 1000 & 1000 \\
\hline
\end{tabular}

Half of the nodes on the upper face of the cylinder were displaced in order to create a complex deformation field at different material interfaces (Figure 6a).

Using the cylindrical geometry, we created a hexahedral mesh (13161 nodes and 12000 elements) and a tetrahedral mesh (11153 nodes and 60030 elements). The behaviour of the following elements was compared:

1) Fully integrated linear hexahedra, with selectively reduced integration of the volumetric term (Hexa), which should offer a benchmark solution;

2) Standard Averaged Nodal Pressure elements (ANP);

3) Our Improved Averaged Nodal Pressure elements (IANP);

4) Linear standard tetrahedron (Tetra).

All the computations were done using the TLED algorithm. Based on the displacement differences presented in Figure 6, we note that the usage of standard locking tetrahedral elements can lead to errors of up to $3.8 \mathrm{~mm}$ in the deformation field. The use of ANP elements reduces the maximum error to $2.3 \mathrm{~mm}$ while the use of IANP elements leads to a maximum error of $1.5 \mathrm{~mm}$ (all errors are considered relative to the results of the model that uses Hexa elements).

The reaction forces computed on the displaced face are presented in Figure 7. The results obtained using the IANP elements are the closest to the benchmark results given by the Hexa elements. Therefore, the IANP elements offer the best performances both in terms of displacements and reaction forces, while the standard four-noded tetrahedral element offers the worst performance, as expected. 
a)
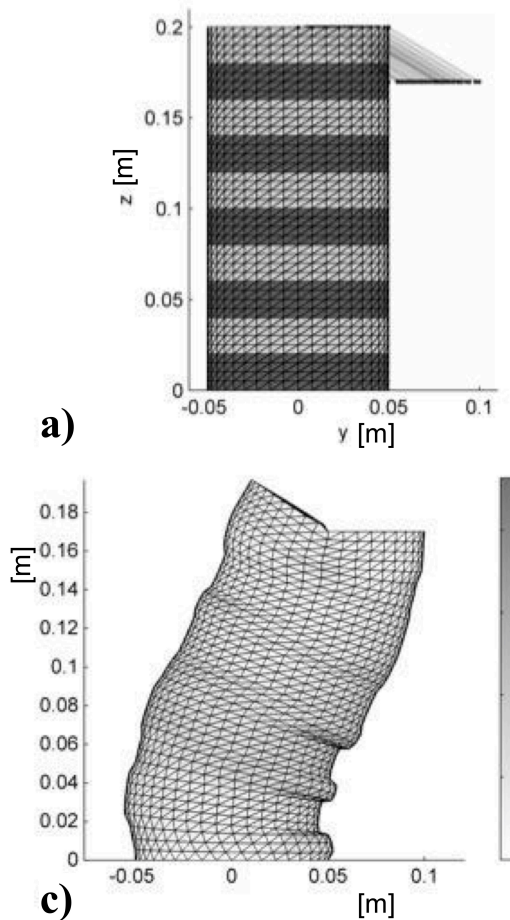
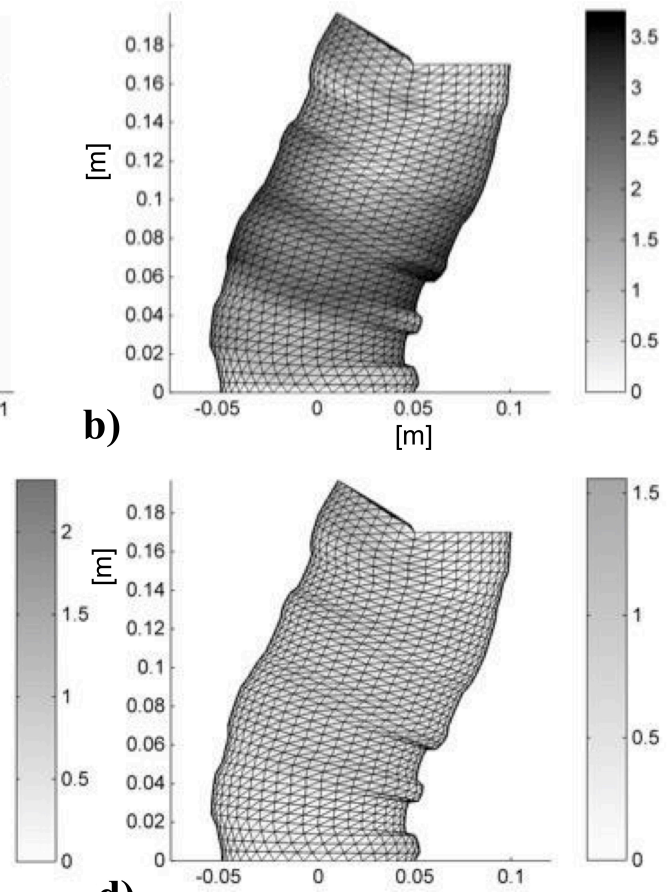

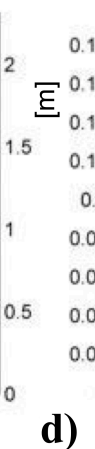

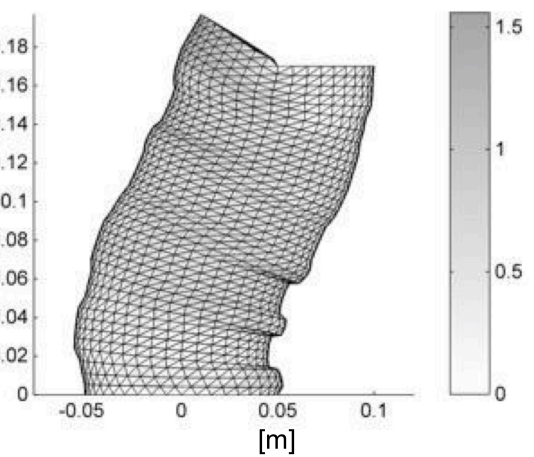

Figure 6 Deformation of a cylinder made out of sections with different material properties. a) The undeformed configuration and the nodal displacements applied. The color bars show the difference in positions of the surface nodes, in mm, between the models using hexahedral elements and models using b) locking tetrahedral elements c) ANP elements and d) IANP elements. Adapted from Joldes et al. [38]. 
a)

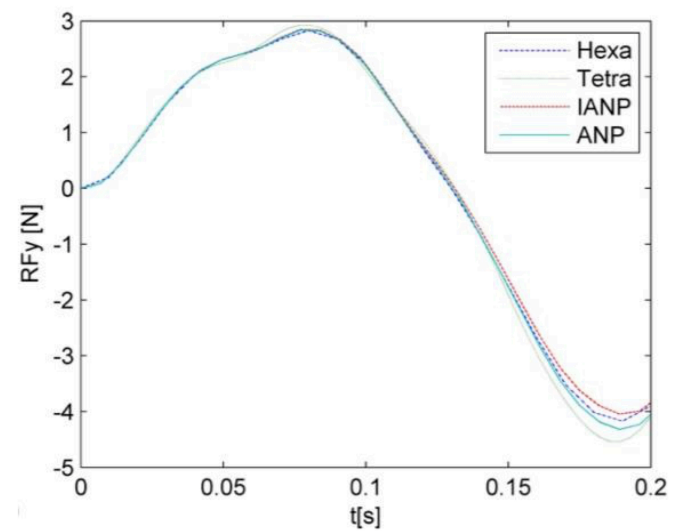

b)

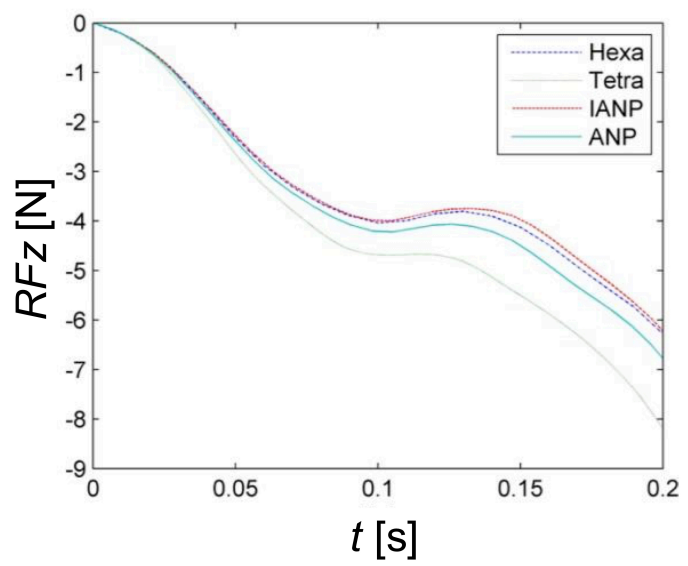

Figure 7 Results obtained using the cylinder model shown in Figure 6: reaction forces on the displaced face a) in the y direction b) in the $\mathrm{z}$ direction. Adapted from Joldes et al. [38].

\subsection{Dynamic Relaxation - steady state computation}

We use this verification example to demonstrate the accuracy of our steady state computation method. For an ellipsoid having approximately the size of the brain, we fixed a set of nodes (at the bottom) 
and displaced another set of nodes (at the top) in order to obtain a deformation field similar to what happens in brain shift. The mesh was created using hexahedral elements and has 2200 elements and 2535 nodes. We used an almost incompressible neo-Hookean material model and a large displacement value $(2 \mathrm{~cm})$.

We performed the displacement computation first by using our algorithm and second by using ABAQUS finite element code [60]. For computational efficiency we use under-integrated hexahedral elements with the hourglass control implemented based on the relations presented in [69]. In ABAQUS, we used hybrid displacement-pressure hexahedral elements, which are the "gold standard" for almost incompressible materials. We used the non-linear static solver from ABAQUS with the default configuration and assumed that the simulation using this solver provides the accurate results.

The error distribution (absolute difference in nodal position between the two simulations) is presented in Figure 8. The maximum error magnitude of $0.6 \mathrm{~mm}$ is obtained at the edge of the displaced area and it is mainly an artifact of using under-integrated elements. Nevertheless, the average error is $0.025 \mathrm{~mm}$ which demonstrates that our simulation results are more than acceptable (as the error is much smaller than the accuracy of image-guided neurosurgery and resolution of neuroimages).

Analysis of the convergence behaviour of adaptive Dynamic Relaxation algorithm when computing deformations of the ellipsoid shown in Figure 8 confirms that the spectral radius determined using the adaptive procedure described in Equations (24) to Eq. (28), leads to increased convergence towards the steady state solution (Figure 9) [42]. This ensures a shorter computation time. 


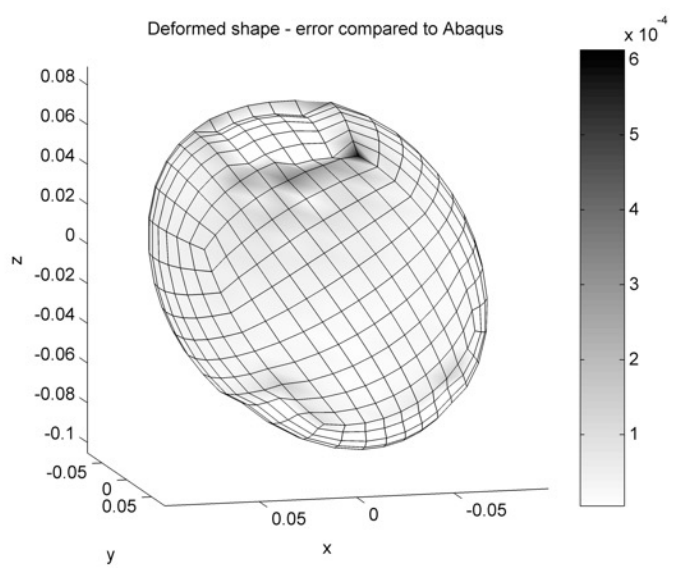

Figure 8 Modelling of indentation of an ellipsoid with the constitutive properties similar to the brain tissue. The figure shows absolute difference (grey-scale coded) in nodal positions between our algorithm and ABAQUS. Dimensions are in meters. Copied from Joldes et al. [40].

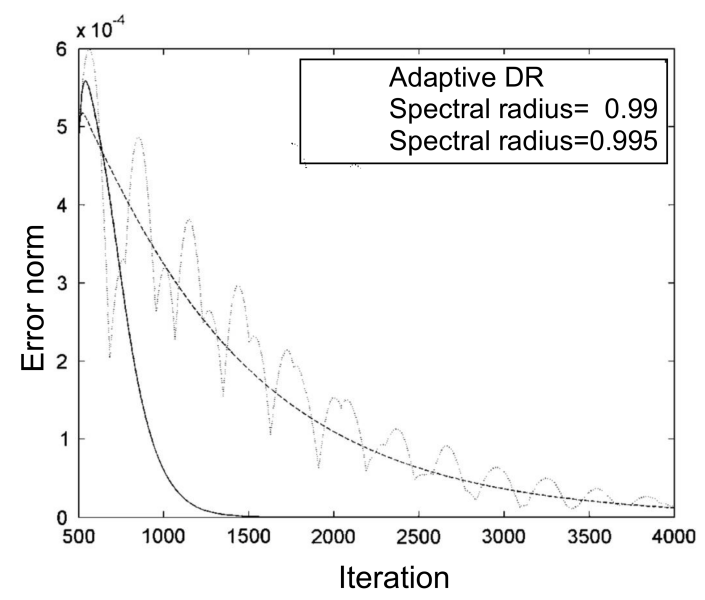

Figure 9 Convergence behaviour of the adaptive Dynamic Relaxation DR algorithm [42] when modelling indentation of the ellipsoid with the constitutive properties similar to the brain tissue (the ellipsoid is shown in Figure 8). The adaptive parameter estimation facilitates faster convergence towards steady state solution than for the Dynamic Relaxation with fixed parameters [40]. Note also the oscillations (underdamped solution) caused by selecting too large spectral radius of 0.995 due to underestimation of the minimum eigenvalue in Equation 20. Adapted from Joldes et al. [42]. 


\subsection{Brain-skull interface - contact algorithm}

In order to assess the performance of our brain-skull interface algorithm, we performed simulations using our implementation of the contact algorithm (combined with Dynamic Relaxation as a solution method) and the commercial explicit dynamics finite element solver LS-DYNA [5], and compared the results. The same loading conditions and material models were used for both solvers. The loading consisted of displacements applied to the nodes in the craniotomy area using a smooth loading curve - a 3-4-5 polynomial that ensures zero velocity and acceleration at the start and end of loading [70]. NeoHookean constitutive models were used for the brain and tumor tissues, and a linear elastic model was used for the ventricles. In order to obtain the steady state solution, the oscillations were damped using both mass and stiffness proportional damping in LS-DYNA.

In a first simulation experiment, we displaced an ellipsoid (made of a hyperelastic neo-Hookean material) with the approximate size of a brain inside another ellipsoid simulating the skull. The maximum displacement applied was $40 \mathrm{~mm}$. The average difference in the nodal displacement field between our simulation and the LS-DYNA simulation was less than $0.12 \mathrm{~mm}$ (Figure 10a).

In the second simulation experiment, we performed the registration of a patient-specific brain shift. The LS-DYNA simulations for this case have been done previously and the results were found to agree well with the real deformations [71]. We performed the same simulations using Dynamic Relaxation and our contact algorithm. The average difference in the nodal displacement field was less than $0.2 \mathrm{~mm}$ (Figure 10b). 

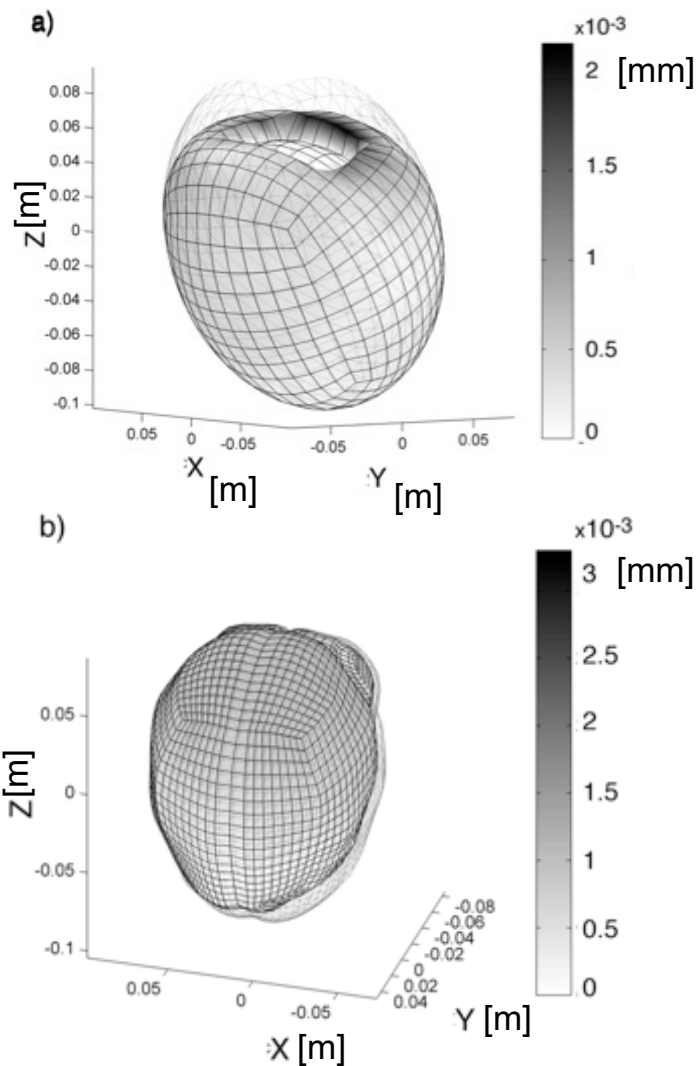

Figure 10 Displacement differences (in millimeters) between our results and LS-DYNA simulations are presented using color codes. The transparent mesh is the master contact. a) Indentation of an ellipsoid (the ellipsoid model is shown in Figure 8); b) Brain model (computation of the brain deformations due to craniotomy induced brain shift). Adapted from Jolde et al. [61].

\section{Conclusions}

Modelling of the brain for injury simulation and computer-assisted neurosurgery is a non-linear problem of continuum mechanics and involves large deformations, very large strains (over 0.8 in compression during needle insertion [21]), non-linear material models, complex loading and boundary conditions, and complex geometry. Various 
finite element (FE) algorithms have been applied for solving this problem.

Modelling of the brain for injury simulation has been often conducted with the goal of using numerical surrogates of the human head in design of countermeasures for traumatic brain injury mitigation [24]. Such modelling has been almost exclusively conducted using non-linear explicit dynamics (i.e. utilising explicit time stepping, referred to in the literature also as explicit time integration $[3,4]$ ) finite element algorithms implemented in commercial finite element codes that are routinely used in the automotive industry for transient dynamics problems involving rapid (impact-type) loading such as car structure responses during collision and sheet metal forming.

However, the computational efficiency of the algorithms available in commercial finite element codes is insufficient for computerintegrated neurosurgery where the solution needs to be provided within the real-time constraints of neurosurgery. This led to development of the specialised non-linear finite element algorithms aiming at satisfying these constraints. We advocate application of non-linear finite element algorithms utilising explicit time stepping (and therefore requiring no iteration for non-linear problems) and Total Lagrangian incremental formulation of continuum mechanics (as it allows precomputing of the derivatives with respect to the spatial coordinates):

- Total Lagrangian explicit dynamics (TLED) finite element algorithm for time accurate solution for surgery simulation;

- Dynamic Relaxation (DR) Total Lagrangian algorithm for computing steady-state deformations for neurosurgery modelling.

For hardware-based increase of computation speed, we propose the implementation of these algorithms on graphics processing units (GPUs) by using a GPU as a coprocessor for the computer central processing unit (CPU). It has been shown in Joldes et al. [64] that the implementation of the finite element Dynamic Relaxation algorithm on NVIDIA Tesla C870 GPU performs 2000 iterations of the brain shift simulation in under $2 \mathrm{~s}$, offering real-time computation capabilities at a fraction of a traditional supercomputer or PC cluster cost. It can be expected that for newer generation of GPUs this already excellent performance would appreciably improve due to significant increase in the number of streaming processor cores (e.g. NVIDIA 
Tesla C870 GPU had 128 cores while NVIDIA Quadro GV100 GPU released in June 2017 has 5120 cores $[62,72]$ ) and available memory (with 32 GB memory for NVIDIA Quadro GV100) [62, 72]).

Application of even most efficient finite element algorithms in surgery simulation and neurosurgery modelling is limited by time consuming generation of patient-specific finite element meshes [73] and deterioration of the solution accuracy when the elements undergo distortion induced by large deformations. As a solution for overcoming these limitations we advocate meshless algorithms in which the computational grid has the form of a "cloud" of points [74-77]. Such algorithms are discussed in Chapter 11 .

\section{Acknowledgements}

This Chapter uses the published results of research supported by the funding from the Australian Government through the Australian Research Council ARC Australian Research Council ARC (grants DP0343112, DP0664534, DP1092893, DP160100714 and LX0560460), National Health and Medical Research Council NHMRC (project grants no. APP1006031 and APP 1144519) and National Institutes of Health NIH (grant 1-R03-CA126466-01A1).

We thank our collaborators Dr Ron Kikinis and Dr Simon K. Warfield of Harvard Medical School (Boston, MA, USA), and Dr Kiyoyuki Chinzei and Dr Toshikatsu Washio of Surgical Assist Technology Group of AIST (Tsukuba, Japan) for help in various aspects of this work.

\section{References}

1. Fressmann, D., Munz, T., Graf, O., Schweizerhof, K.: FE human modelling in crash - aspects of the numerical modelling and current applications in the automotive industry. In: DYNAmore GmbH, Frankthenhal, Germany, pp. FI-23 - F-I-34 (2007) 
2. Takhounts, E.G., Eppinger, R.H., Campbell, J.Q., Tannous, R.E., Power, E.D., Shook, L.S.: On the development of the SIMon finite element head model. Stapp Car Crash Journal 47, 107-133 (2003)

3. Belytschko, T., Liu, W.K., Moran, B.: Nonlinear Finite Elements for Continua and Structures. John Wiley\&Sons, Chichester (2006)

4. Bathe, K.-J.: Finite Element Procedures. Prentice-Hall (1996)

5. Livermore Software Technology Coporation LSTC: LS-DYNA Theory Manual, http://www.lstc.com/download/manuals (2018)

6. Pacific Engineering Systems International (ESI): PAM-SAFE http://www.esi.com.au/Software/PAM-SAFE.html (2018)

7. Altair: RADIOSS Technical Papers, https://altairhyperworks.com/ResourceLibrary.aspx?category=Technical\%20 Papers\&altair products=RADIOSS (2018)

8. Dassault Systèmes Simulia Corporation: ABAQUS Online Documentation: Version 6.14 (2014)

9. Baumann, R., Glauser, D., Tappy, D., Baur, C., Clavel, R.: Force feedback for virtual reality based minimally invasive surgery simulator. Studies in Health Technology and Informatics 29, 564-579 (1996)

10. Cover, S.A., Ezquerra, N.F., O'Brien, J.F., Rowe, R., Gadacz, T., Palm, E.: Interactively deformable models for surgery simulation. Computer Graphics and Applications, IEEE 13, 68-75 (1993)

11. Kuhnapfel, U., Çakmak, H.K., Maaß, H.: Endoscopic surgery training using virtual reality and deformable tissue simulation. Computers \& Graphics 24, 671-682 (2000)

12. Nimura, Y., Di Qu, J., Hayashi, Y., Oda, M., Kitasaka, T., Hashizume, M., Misawa, K., Mori, K.: Pneumoperitoneum simulation based on mass-springdamper models for laparoscopic surgical planning. Journal of Medical Imaging 2, 044004 (2015)

13. Cotin, S., Delingette, H., Ayache, N.: A hybrid elastic model for real-time cutting, deformations, and force feedback for surgery training and simulation. The Visual Computer 16, 437-452 (2000)

14. Bro-Nielsen, M.: Finite element modeling in surgery simulation. Proceedings of the IEEE 86, 490-503 (1998)

15. Bro-Nielsen, M., Cotin, S.: Real-time volumetric deformable models for surgery simulation using finite elements and condensation. Computer Graphics Forum 15, 57-66 (1996) 
16. DiMaio, S.P., Salcudean, S.E.: Interactive simulation of needle insertion models. IEEE Transactions on Biomedical Engineering 52, 1167-1179 (2005)

17. Warfield, S.K., Talos, F., Tei, A., Bharatha, A., Nabavi, A., Ferrant, M., Black, P.M., Jolesz, F.A., Kikinis, R.: Real-time registration of volumetric brain MRI by biomechanical simulation of deformation during image guided neurosurgery. Computing and Visualization in Science 5, 3-11 (2002)

18. Dumpuri, P., Thompson, R.C., Cao, A., Ding, S., Garg, I., Dawant, B.M., Miga, M.I.: A fast and efficient method to compensate for brain shift for tumor resection therapies measured between pre-operative and post-operative tomograms. IEEE Transactions on Bio-medical Engineering 57, 1285-1296 (2010)

19. Dumpuri, P., Thompson, R.C., Dawant, B.M., Cao, A., Miga, M.I.: An atlasbased method to compensate for brain shift: Preliminary results. Medical Image Analysis 11, 128-145 (2007)

20. Roberts, D.W., Hartov, A., Kennedy, F.E., Miga, M.I., Paulsen, K.D.: Intraoperative brain shift and deformation: A quantitative analysis of cortical displacement in 28 cases. Neurosurgery 43, 749-758 (1998)

21. Wittek, A., Dutta-Roy, T., Taylor, Z., Horton, A., Washio, T., Chinzei, K., Miller, K.: Subject-specific non-linear biomechanical model of needle insertion into brain. Computer Methods in Biomechanics and Biomedical Engineering 11, 135-146 (2008)

22. Wittek, A., Hawkins, T., Miller, K.: On the unimportance of constitutive models in computing brain deformation for image-guided surgery. Biomechanics and Modeling in Mechanobiology 8, 77-84 (2009)

23. Belytschko, T.: A survey of numerical methods and computer programs for dynamic structural analysis. Nuclear Engineering and Design 37, 23-34 (1976)

24. Yang, K.H.: Basic Finite Element Method as Applied to Injury Biomechanics. Academic Press (2018)

25. Cook, R.D., Malkus, D.S., Plesha, M.E.: Finite elements in dynamics and vibrations. Concepts and Applications of Finite Element Analysis, pp. 367428. John Wiley \& Sons, New York (1989)

26. Hughes T. J. R.: Analysis of Transient Algorithms With Particular Reference To Stability Behavior. In: Belytschko, T., Hughes, T.J.R. (eds.) Computational Methods for Transient Analysis, vol. 1, pp. 67-155. North-Holland, Amsterdam (1983)

27. Belytschko, T.: An Overview of Semidiscretization and Time Integration Procedures. In: Belytschko, T., Hughes, T.J.R. (eds.) Computational Methods for Transient Analysis, vol. 1, pp. 1-66. North-Holland, Amsterdam (1983)

28. Olovsson, L., Simonsson, K., Unosson, M.: Selective mass scaling for explicit finite element analyses. International Journal for Numerical Methods in Engineering 63, 1436-1445 (2005) 
29. Pan, F., Zhu, J., Helminen, A.O., Vatanparast, F.: Three point bending analysis of a mobile phone using LS-DYNA explicit integration method. In: Mindle, W.L. (ed.) 9th International LS-DYNA Users Conference. Livermore Software Technology Corporation, Dearborn, MI, USA (2006)

30. Majumder, S., Roychowdhury, A., Subrata, P.: Three-dimensional finite element simulation of pelvic fracture during side impact with pelvis-femursoft tissue complex. International Journal of Crashworthiness 13, 313-329 (2008)

31. Cocchetti, G., Pagani, M., Perego, U.: Selective mass scaling and critical timestep estimate for explicit dynamics analyses with solid-shell elements. Computers \& Structures 127, 39-52 (2013)

32. Flanagan, D.P., Belytschko, T.: A uniform strain hexahedron and quadrilateral with orthogonal hourglass control. International Journal for Numerical Methods in Engineering 17, 679-706 (1981)

33. Cifuentes, A.O., Kalbag, A.: A performance study of tetrahedral and hexahedral elements in 3-D finite element structural analysis. Finite Elements in Analysis and Design 12,313-318 (1992)

34. Cook, R.D., Malkus, D.S., Plesha, M.E.: Concepts and Applications of Finite Element Analysis. John Wiley \& Sons, New York (1989)

35. Hughes, T.J.R.: The Finite Element Method: Linear Static and Dynamic Finite Element Analysis. Dover Publications, Mineola (2000)

36. Rojek, J., Oñate, E., Postek, E.: Application of explicit FE codes to simulation of sheet and bulk metal forming processes. Journal of Materials Processing Technology 80-81, 620-627 (1998)

37. Miller, K., Joldes, G., Lance, D., Wittek, A.: Total Lagrangian explicit dynamics finite element algorithm for computing soft tissue deformation. Commun. Numer. Methods Eng. 23, 121-134 (2007)

38. Joldes, G.R., Wittek, A., Miller, K.: Non-locking tetrahedral finite element for surgical simulation. Communications in Numerical Methods in Engineering 25, 827-836 (2008)

39. Underwood, P.: Dynamic Relaxation. In: Belytschko, T., Hughes, T.J.R. (eds.) Computational Methods for Transient Analysis, vol. 1, pp. 245-265. NewHolland, Amsterdam (1983)

40. Joldes, G.R., Wittek, A., Miller, K.: Computation of intra-operative brain shift using dynamic relaxation. Computer Methods in Applied Mechanics and Engineering 198, 3313-3320 (2009)

41. Isaacson, E.: Analysis of numerical methods. Wiley, New York (1966) 
42. Joldes, G.R., Wittek, A., Miller, K.: An adaptive dynamic relaxation method for solving nonlinear finite element problems. Application to brain shift estimation. International Journal for Numerical Methods in Biomedical Engineering 27, 173-185 (2011)

43. Miller, K.: Biomechanics of the Brain for Computer Integrated Surgery. Publishing House of Warsaw University of Technology, Warsaw (2002)

44. Miller, K., Chinzei, K.: Mechanical properties of brain tissue in tension. Journal of Biomechanics 35, 483-490 (2002)

45. Miller, K., Chinzei, K., Orssengo, G., Bednarz, P.: Mechanical properties of brain tissue in-vivo: experiment and computer simulation. Journal of Biomechanics 33, 1369-1376 (2000)

46. Miller, K.: Constitutive modelling of abdominal organs. Journal of Biomechanics 33, 367-373 (2000)

47. Miller, K., Chinzei, K.: Constitutive modelling of brain tissue; Experiment and Theory. Journal of Biomechanics 30, 1115-1121 (1997)

48. Bilston, L.E., Liu, Z., Phan-Tien, N.: Linear viscoelastic properties of bovine brain tissue in shear. Biorheology 34, 377-385 (1997)

49. Margulies, S.S., Thibault, L.E., Gennarelli, T.A.: Physical model simulations of brain injury in the primate. Journal of Biomechanics 23, 823-836 (1990)

50. Bonet, J., Burton, A.J.: A simple averaged nodal pressure tetrahedral element for incompressible and nearly incompressible dynamic explicit applications. Communications in Numerical Methods in Engineering 14, 437-449 (1998)

51. Bonet, J., Marriott, H., Hassan, O.: An averaged nodal deformation gradient linear tetrahedral element for large strain explicit dynamic applications. Communications in Numerical Methods in Engineering 17, 551-561 (2001)

52. Zienkiewicz, O.C., Rojek, J., Taylor, R.L., Pastor, M.: Triangles and Tetrahedra in Explicit Dynamic Codes for Solids. International Journal for Numerical Methods in Engineering 43, 565-583 (1998)

53. Dohrmann, C.R., Heinstein, M.W., Jung, J., Key, S.W., Witkowski, W.R.: Node-based uniform strain elements for three-node triangular and four-node tetrahedral meshes. International Journal for Numerical Methods in Engineering 47, 1549-1568 (2000)

54. Joldes, G.R., Wittek, A., Miller, K.: Non-locking tetrahedral finite element for surgical simulation. Commun. Numer. Methods Eng. 25, 827-836 (2009)

55. Joldes, G.R., Wittek, A., Miller, K.: An efficient hourglass control implementation for the uniform strain hexahedron using the Total Lagrangian formulation. Commun. Numer. Methods Eng. 23, 315-323 (2008)

56. Hallquist, J.O., Goudreau, G.L., Benson, D.J.: Sliding interfaces with contactimpact in large-scale Lagrangian computations. Computer Methods in Applied Mechanics and Engineering 51, 107-137 (1985) 
57. Doghri, I., Muller, A., Taylor, R.L.: A general three-dimensional contact procedure for implicit finite element codes. Engineering Computations 15, 233-259 (1998)

58. Stewart, J.R., Gullerud, A.S., Heinstein, M.W.: Solution verification for explicit transient dynamics problems in the presence of hourglass and contact forces. Computer Methods in Applied Mechanics and Engineering 195, 14991516 (2006)

59. Sauvé, R.G., Morandin, G.D.: Simulation of contact in finite deformation problems - algorithm. International Journal of Mechanics and Materials in Design 1, 287-316 (2004)

60. Dassault Systèmes Simulia Corporation: ABAQUS Theory Guide: Version 6.14 (2014)

61. Joldes, G., Wittek, A., Miller, K., Morriss, L.: Realistic and efficient brainskull interaction model for brain shift computation. In: Computational Biomechanics for Medicine III, International Conference on Medical Image Computing and Computer-Assisted Intervention MICCAI 2008, pp. 95-105. Medical Image Computing and Computer-Assisted Intervention, http://www.cbm.mech.uwa.edu.au/CBM2008/, (2008)

62. NVIDIA: NVIDIA's Next Generation CUDA Compute Architecture: Fermi, http://www.nvidia.com/content/PDF/fermi white papers/NVIDIA Fermi C ompute Architecture Whitepaper.pdf (2009)

63. Khronos: Open CL https://www.khronos.org/opencl/ (2018)

64. Joldes, G.R., Wittek, A., Miller, K.: Real-Time Nonlinear Finite Element Computations on GPU - Application to Neurosurgical Simulation. Computer Methods in Applied Mechanics and Engineering 199, 3305-3314 (2010)

65. Comas, O.: Real-time Soft Tissue Modelling on GPU for Medical Simulation. Doctoral School of Engineering Science, PhD Thesis. Université des Sciences et Technologie de Lille Lille, France (2011)

66. Courtecuisse, H., Allard, J., Kerfriden, P., Bordas, S.P.A., Cotin, S., Duriez, C.: Real-time simulation of contact and cutting of heterogeneous soft-tissues. Medical Image Analysis 18, 394-410 (2014)

67. The American Society of Mechanical Engineers ASME, Standards Committee on Verification and Validation in Computational Solid Mechanics PTC 60/V\&V 10: Guide for Verification and Validation in Computational Solid Mechanics. http://cstools.asme.org/csconnect/pdf/CommitteeFiles/24816.pdf (2006)

68. Babuska, I., Oden, J.T.: Verification and validation in computational engineering and science: basic concepts. Computer Methods in Applied Mechanics and Engineering 193, 4057-4066 (2004) 
69. Joldes, G.R., Wittek, A., Miller, K.: An Efficient Hourglass Control Implementation for the Uniform Strain Hexahedron Using the Total Lagrangian Formulation. Communications in Numerical Methods in Engineering DOI: 10.1002/cnm.1034 (2007)

70. Waldron, K.J., Kinzel, G.L.: Kinematics, dynamics, and design of machinery. John Wiley, New York (1999)

71. Wittek, A., Miller, K., Kikinis, R., Warfield, S.K.: Patient-specific model of brain deformation: Application to medical image registration. Journal of Biomech. 40, 919-929 (2007)

72. NVIDIA: Quadro on Desktop Workstations https://www.nvidia.com/enus/design-visualization/quadro-desktop-gpus/

73. Wittek, A., Grosland, N., Joldes, G., Magnotta, V., Miller, K.: From finite element meshes to clouds of points: A Review of methods for generation of computational biomechanics models for patient-specific applications. Ann Biomed Eng 44, 3-15 (2016)

74. Horton, A., Wittek, A., Joldes, G.R., Miller, K.: A meshless Total Lagrangian explicit dynamics algorithm for surgical simulation. International Journal for Numerical Methods in Biomedical Engineering 26, 977-998 (2010)

75. Miller, K., Horton, A., Joldes, G.R., Wittek, A.: Beyond finite elements: A comprehensive, patient-specific neurosurgical simulation utilizing a meshless method. Journal of Biomechanics 45, 2698 - 2701 (2012)

76. Zhang, G.Y., Wittek, A., Joldes, G.R., Jin, X., Miller, K.: A three-dimensional nonlinear meshfree algorithm for simulating mechanical responses of soft tissue. Engineering Analysis with Boundary Elements 42, 60-66 (2014)

77. Li, M., Miller, K., Joldes, G.R., Kikinis, R., Wittek, A.: Biomechanical model for computing deformations for whole-body image registration: A meshless approach. International Journal for Numerical Methods in Biomedical Engineering 32, e02771-02771-02718 (2016) 